\title{
Skin Fluid or Secretion
}

National Cancer Institute

\section{Source}

National Cancer Institute. Skin Fluid or Secretion. NCI Thesaurus. Code C13409.

Secretions of the skin. 